\title{
A Report of Physiotherapy Rehabilitation in A Case of Post- Surgical Anterior Cruciate Ligament Reconstruction
}

\author{
Simran Jaiswal ${ }^{1}$, Aachal Birelliwar ${ }^{1}$, Neha Chitale ${ }^{2}$, Pratik Phansopkar ${ }^{1}$ \\ ${ }^{1}$ Intern, Ravi Nair Physiotherapy College, DattaMeghe Institute of Medical \\ Sciences, Wardha, Maharashtra, India -442001 \\ ${ }^{2}$ Resident, Department of Musculoskeletal Physiotherapy, Ravi Nair Physiotherapy \\ College, DattaMeghe Institute of Medical Sciences, Wardha, Maharashtra, India -442001 \\ Corresponding author email: drpratik77@gmail.com
}

\section{ABSTRACT}

Anterior Cruciate ligament $(\mathrm{ACL})$ is the most common ligament to get damaged in the knee joint. Knee joint being a dynamic structure requires lot of stabilizing factors to maintain dynamic stability of the joint. ACLhelps to maintain dynamic stability of the knee joint by preventing anterior translation of tibia.ACL and PCLserve as primary rotatory stabilizers for knee joint. This case study reflects on the treatment of a case of ACL reconstruction of the left knee. A 50 years old male who met an accident reported with anterior cruciate ligament tear. Patient had reported with swelling over knee region and he had been treated surgically for anterior cruciate ligament reconstruction. The patient was mesomorphic in nature. Physical therapy rehabilitation protocol mainly focused on strengthening of quadriceps and hamstrings muscle and accordingly provided care in ways including postoperative weight bearing, gait training, improving strength of quadriceps and hamstring muscles. Anterior Cruciate Ligament Tear following accidental trauma. Therapeutic Intervention and Post-operative knee brace was given to the patient after surgery which was locked in slight hyperextension. The patient was made aware of the need to wear the brace even while sleeping. CPM machine was used daily for 3 hours per day. Outpatient physiotherapy was started on post-operative day 10. Basic exercises such as active and passive ROM, strengthening exercise for quadriceps, straight leg raising with brace. After 7th week there was complete recovery in passive range of motion of the knee joint. Early postoperative recovery is ensured withtimely initiation of physiotherapy and the motivation to continue with the physiotherapy.

\section{KEY WORDS: KNEE JOINT, ANTERIOR CRUCIATE LIGAMENT, TEAR, TREATMENT, REHABILITATION.}

\section{INTRODUCTION}

This is a case of 50 years old male, who underwent anterior cruciate ligament reconstruction after an accident. Rehabilitation after anterior cruciate ligament reconstruction (ACL) has shown a fast evolution over 25 years.However, there is scantness of objective and standardized criteria to access properly person's ability

Biosc Biotech Res Comm P-ISSN: 0974-6455 E-ISSN: 2321-4007

\section{crossef}

Identifiers and Pagination

Year: 2021 Vol: 14 No (6) Special Issue

Pages: 116-119

This is an open access article under Creative

Commons License Attribn 4.0 Intl (CC-BY).

DOI: $h t t p: / / d x$.doi.org/10.21786/bbrc/14.6.27 to progress to the last stage of the physical therapy rehabilitation and safely return to their work (Myeret al., 2006).

Physiotherapy is usually prescribed after ACL reconstruction. Physiotherapy goals vary from one person to other. The goals depend on the patient's signs and also their symptoms. Goals also depends upon the time from the operation. One basic goal of the physiotherapy rehabilitation is to improve and increase the strength of the quadriceps muscles. There are many researches which have shown the presence of weakness of quadriceps muscles after the reconstruction of anterior cruciate ligament. Improvement in physical function and also strength of the quadriceps muscles are seen in patients who regularly perform strengthening of the

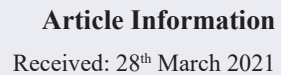

ccepted after revision: $6^{\text {th }}$ May 2021 
quadriceps after ACL reconstruction as a part of their rehabilitation. Basically, strength training exercises of the quadriceps are done (Pivaet al., 2009).The main aim of the anterior cruciate ligament operation and physical therapy rehabilitation is to improve knee function to the maximum and also restoring the knee function as it was before the injury. These aims are usually not achieved. As there is weakness of the quadriceps muscles there is poor functional outcome. Therefore, identification of techniques and to improve strength of the quadriceps after the anterior cruciate ligament injury and reconstruction is of greater clinical interest (Palmieriet al., 2008).

ACL injuries are destructive and damaging because of significant long and short term financial, psychological and functional consequences. It requires long term rehabilitation. Rehabilitation could take at least 6 to 24 months (Tayloret al., 2020).

Patient information: The patient is 50 year, male farmer with normal BMI and left hand dominance complained of dull aching type of pain at the anterior aspect of the knee joint, which aggravates while walking and relieved at rest, it was also associated with swelling.

With these complains the patient visited a nearby hospital where investigation such as X-ray was done. Crepe bandage was applied and medications were given to the patient. He applied crepe bandage for 2 days but there was no relief. Then he visited AVBRH where investigations such as X-ray and MRI were done and the patient was diagnosed with anterior cruciate ligament tear (Grade 3) and advised for surgery. The patient was operated for ACL reconstruction on 22nd October. Arthroscopic ACL reconstruction of left knee was performed. Post operatively, patient was referred to physiotherapy department with the complain of pain, inability to flex knee.

Clinical findings: On post-operative day 1 after the ACL reconstruction, the patient's heart rate was $72 \mathrm{bpm}$, respiratory rate was 20 breaths per minute, blood pressure was 110/80 mmHg. Patient was alert and well oriented with time, place and person. The patient's overall posture was assessed and there were no abnormalities seen. In the affected leg there was local rise in temperature and scars were noted one was $4 \mathrm{~cm}$ medial to tibial tuberosity, other one $1 \mathrm{~cm}$ above patella laterally and the last one $1 \mathrm{~cm}$ below patella laterally.

In supine position the hip was externally rotated, knee 5 degree of flexion, ankle plantarflexed and foot inverted. Pain stated by the patient on NPRS scale was 6/10. In the affected leg measurement of active and passive range of motion (ROM) using goniometer at hip joint were flexion: 110 degrees and passive was 115 degrees. Measurement of active ROM of knee joint was 60 degree and passive was 64 degrees. Measurement of active ROM of ankle dorsiflexors was 18 degree and passive ankle dorsiflexors was 20 degrees. Measurement of active
ROM of ankle plantarflexors was 40 degrees and passive ankle plantarflexors was 45 degrees. In the non-affected leg measurement of active Rom of hip flexion was 115 degree and passive ROM was 120 degrees. Measurement of active ROM of knee flexion was 120 degree and passive ROM was 125 degrees. Measurement of active ROM of ankle plantarflexors was 40 degree and passive ROM of plantarflexors was 45 degrees. Measurement active ROM of ankle dorsiflexors was 18 degrees and Passive ROM of ankle dorsiflexors was 20 degrees.

Girth measurement of the affected and the unaffected leg was done which was equal in both the legs. Limb length discrepancy was noted. True leg length of the affected leg was $83 \mathrm{~cm}$ and the apparent leg length of the affected leg was $95 \mathrm{~cm}$. True leg length of unaffected leg was $36 \mathrm{~cm}$ and apparent leg length of unaffected leg was $97 \mathrm{~cm}$. Gait of the patient was normal. Decreased knee flexion while walking was noticed. Strength and ROM of upper extremity of the patient was adequate to perform the activities of daily living. The uninvolved leg was functional. As per standard International Classification of Functioning, Disability and Health (ICF) there was a problem in an affected leg as he was unable to walk properly, there was pain after certain range, stability of joint was affected and there was also decreased strength of muscles.

Therapeutic Intervention: Post-operative knee brace was given to the patient after surgery which was locked in slight hyperextension. Instruction was given to the patient to wear the brace throughout the day and only remove it at the time of taking bath or at the time of physical therapy. The patient was made aware of the need to wear the brace even while sleeping. CPM machine was used daily for 3 hours per day and the ROM was set from 0 to 25 degrees and the patient was asked to progress the movement by 5 to 10 degrees of flexion if the pain permits. Patient used bilateral axillary crutch and was restricted to toe touch weight bearing. Gait training of the patient was done, weight bearing was avoided.

Outpatient physiotherapy was started on post-operative day 10. Basic exercises such as active and passive ROM, strengthening exercise for quadriceps, straight leg raising with brace. The patient was advised to keep his leg elevated for 15 minutes in an hour. On the 3rd week after the operation there was improvement in passive range of motion of the knee joint. After 7th week there was complete recovery in passive range of motion of the knee joint. Increased strength was seen in the quadriceps muscle and the patient was able to perform straight leg raising. There was adequate strength in the knee so the patient was able to walk without crutch.

Follow up and outcome: The patient was able to walk without any support or assistance by the end of the session. He was also independent. The patient was willing to do physiotherapy and was well motivated and did whatever was asked him to do. Home exercises program was also tough to the patient. She did the home exercises 
regularly and also visited the department regularly. the patient was psychologically fit which was a positive factor which helped to treat him properly.

\section{DISCUSSION}

Anterior Cruciate ligament is the most common ligament to get damaged in the knee joint. Knee joint being a dynamic structure requires lot of stabilizing factors to maintain dynamic stability of the joint. ACL and PCL are mainly responsible for the rotatory stability of the joint of which ACL is prevents the anterior translation of the knee joint. In this study, reconstruction was done using arthroscopic method. The primary aim was patient education, prevention of secondary complication and maintenance as well as strengthening of quadriceps and hamstrings muscles. The study shows that after undergoing anterior cruciate ligament reconstruction patient may require few months to achieve normal quadriceps muscle function. One of the essential outcome indicators is an increase in the strength of the quadriceps muscles.

Using weight bearing exercise of the lower limb improves the functional outcome better than non-weight bearing exercise (Greenberget al., 2012; Risberget al., 1999; Baiset al., 2020). Few of the related studies were reviewed (Waneet al., 2020; Murrayet al., 2020; Murrayet al., 2019; Voset al., 2019; Wanget al., 2020; and Lozanoet al., 2020). Studies on anterior cruciate ligament injury and knee joint were reported by Risaldar et. al., 2020; Mohabey et. al.,2020 and Vaidya et. al., 2020). Bari et. al., 2014 reported on evaluation of MRI versus arthroscopy in anterior cruciate ligament and meniscal injuries (Darware et. al., 2020)reported a case of physiotherapy rehabilitation accelerating the recovery of older patient with anterior cruciate ligament reconstruction (Nikose et. al., 2020). Some of the interesting studies were reported by (Maniar et. al., 2012; Bhandakkar et. al., 2020).

\section{CONCLUSION}

After Physiotherapy intervention significant improvement was seen in the strength of both hamstrings and quadriceps. The patient was willing to do physiotherapy and was well motivated.The patient was able to walk without any support or assistance by the end of the session. He was also independent.

Author's contribution: All authors made best contribution for the concept, assessment and evaluation, data acquisition and analysis and interpretation of the data

Ethical clearance: The institution ethical committee clearance is obtained.

\section{Conflict of interest: Nil}

\section{Funding Support: Nil}

\section{REFERENCES}

Bais, A., Bawiskar, D., Naqvi, W.M. and Sahu, A., 2020. A case study on the impact of physiotherapy on unilateral foot drop after lumbar fusion and discectomy. Medical Science, 24(103), pp.1773-1779.

Bari, A.A., Kashikar, S.V., Lakhkar, B.N. and Ahsan, M.S., 2014. Evaluation of MRI versus arthroscopy in anterior cruciate ligament and meniscal injuries. Journal of clinical and diagnostic research: JCDR, 8(12), p.RC14. Bhandakkar, P.A., Naqvi, W. and Burhani, T.S., 2020. Impact of Physiotherapy Rehabilitation on Patients with Bilateral Osteoarthritis Knee Pain--A Case Report. Journal of Evolution of Medical and Dental Sciences, 9(32), pp.2316-2318.

Darware, M., Ghordadekar, D., Dhole, R. and Noman, O., 2020. A Case Report On Physiotherapy Rehabilitation Leading To Early Recovery After Hysterectomy And Prevention Of Postoperative Complications. European Journal of Molecular \& Clinical Medicine, 7(11), pp.3304-3311.

Greenberg, E.M., Albaugh, J., Ganley, T.J. and Lawrence, J.T.R., 2012. Rehabilitation considerations for all epiphyseal ACL reconstruction. International journal of sports physical therapy, 7(2), p.185.

Latchoumi, T.P., Ezhilarasi, T.P. and Balamurugan, K., 2019. Bio-inspired weighed quantum particle swarm optimization and smooth support vector machine ensembles for identification of abnormalities in medical data. SN Applied Sciences, 1(10), pp.1-10.

Lozano, R., Fullman, N., Mumford, J.E., Knight, M., Barthelemy, C.M., Abbafati, C., Abbastabar, H., AbdAllah, F., Abdollahi, M., Abedi, A. and Abolhassani, H., 2020. Measuring universal health coverage based on an index of effective coverage of health services in 204 countries and territories, 1990-2019: a systematic analysis for the Global Burden of Disease Study 2019. The Lancet, 396(10258), pp.1250-1284.

Maniar, R.N., Singhi, T., Rathi, S.S., Baviskar, J.V. and Nayak, R.M., 2012. Surgical technique: lateral retinaculum release in knee arthroplasty using a stepwise, outside-in technique. Clinical Orthopaedics and Related Research ${ }^{\circledR}$, 470(10), pp.2854-2863.

Mohabey, A., Gupta, S., Gawande, V. and Saoji, K., 2020. A Study on Correlation of Magnetic Resonance Imaging and Arthroscopy in Evaluation of Anterior Cruciate Ligament Injury in Cases of Acute Traumatic Haemarthrosis of Knee: A Prospective Study. Int J Cur Res Rev| Vol, 12(14).

Murray, C.J., Abbafati, C., Abbas, K.M., Abbasi, M., Abbasi-Kangevari, M., Abd-Allah, F., Abdollahi, M., Abedi, P., Abedi, A., Abolhassani, H. and Aboyans, V., 2020. Five insights from the global burden of disease study 2019. The Lancet, 396(10258), pp.1135-1159.

Murray, C.J., Aravkin, A.Y., Zheng, P., Abbafati, C., Abbas, K.M., Abbasi-Kangevari, M., Abd-Allah, F., Abdelalim, A., Abdollahi, M., Abdollahpour, I. and Abegaz, K.H., 2020. Global burden of 87 risk factors in 204 countries and territories, 1990-2019: a systematic analysis for the Global Burden of Disease Study 2019. 
The Lancet, 396(10258), pp.1223-1249.

Myer, G.D., Paterno, M.V., Ford, K.R., Quatman, C.E. and Hewett, T.E., 2006. Rehabilitation after anterior cruciate ligament reconstruction: criteria-based progression through the return-to-sport phase. Journal of Orthopaedic \& Sports Physical Therapy, 36(6), pp.385-402.

Nikose, S.S., Nikose, D., Jain, S., Kekatpure, A., Saoji, K., Chaudhary, R. and Pisulkar, G., 2021. Determinants of regeneration and strength of hamstrings after anterior cruciate ligament reconstruction-fate of hamstring tendon. International Orthopaedics, pp.1-10.

Palmieri-Smith, R.M., Thomas, A.C. and Wojtys, E.M., 2008. Maximizing quadriceps strength after ACL reconstruction. Clinics in sports medicine, 27(3), pp.405424.

Piva, S.R., Childs, J.D., Klucinec, B.M., Irrgang, J.J., Almeida, G.J. and Fitzgeiald, G.K., 2009. Patella fracture during rehabilitation after bone-patellar tendon-bone anterior cruciate ligament reconstruction: 2 case reports. Journal of orthopaedic \&t sports physical therapy, 39(4), pp.278-286.

Risaldar, P., Raut, A., Bawiskar, D. and Naqvi, W.M., 2020. Impact of Physiotherapy rehabilitation program on postoperative ACL tear patient on prognosis leading to maintain consistency in sport. Int. J. Res. Pharm. Sci, 11, pp.4821-4825.

Risberg, M.A., Holm, I., Tjomsland, O., Ljunggren, E. and Ekeland, A., 1999. Prospective study of changes in impairments and disabilities after anterior cruciate ligament reconstruction. Journal of Orthopaedic \&
Sports Physical Therapy, 29(7), pp.400-412.

Taylor, J.B., Owen, E. and Ford, K.R., 2020. Incorporating Workload Measures Into Rehabilitation After Anterior Cruciate Ligament Reconstruction: A Case Report. International Journal of Sports Physical Therapy, 15(5), p.823.

Vaidya, S.V., Dhongade, H.S., Mahajan, S.M. and MK, A., 2020. Evaluation of Anterior and Posterior Cruciate Ligament Injuries of the Knee on Magnetic Resonance Imaging: A Cross-sectional Study. Int J Cur Res Rev| Vol, 12(14), p.105.

Vos, T., Lim, S.S., Abbafati, C., Abbas, K.M., Abbasi, M., Abbasifard, M., Abbasi-Kangevari, M., Abbastabar, H., Abd-Allah, F., Abdelalim, A. and Abdollahi, M., 2020. Global burden of 369 diseases and injuries in 204 countries and territories, 1990-2019: a systematic analysis for the Global Burden of Disease Study 2019. The Lancet, 396(10258), pp.1204-1222.

Wane, M., Naqvi, W.M., Vaidya, L. and Kumar, K., 2020. Kinesiophobia in a Patient With Postoperative Midshaft Fracture: A Case Report of Its Impact on Rehabilitation in a 16-Year-Old Girl. Cureus, 12(11).

Wang, H., Abbas, K.M., Abbasifard, M., AbbasiKangevari, M., Abbastabar, H., Abd-Allah, F., Abdelalim, A., Abolhassani, H., Abreu, L.G., Abrigo, M.R. and Abushouk, A.I., 2020. Global age-sex-specific fertility, mortality, healthy life expectancy (HALE), and population estimates in 204 countries and territories, 1950-2019: a comprehensive demographic analysis for the Global Burden of Disease Study 2019. The Lancet, 396(10258), pp.1160-1203. 